place to start if you are looking for an introduction to the next frontier in neuroscience. Many of these odorant receptor genes are located in the telomeric region of almost all chromosomes and are seen in abundance on chromosomes 7, 16, and 17. A number of our patients are now being identified with telomeric deletions. Is there a connection between odor receptor deletions and the developmental phenotype of these patients?

If you are going to be around Nice anytime soon, you might want to plan a day trip to Le Manie, $80 \mathrm{~km}$ east along the coast. It has been described as being one of the most scented regions of our world.

\title{
Molecular bases of mental retardation
}

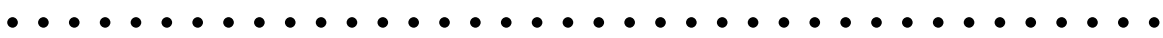
Mental Retardation and Developmental Delay - Genetic and Epigenetic Factors

Moyra Smith (ed)

Oxford University Press, 2006. 317pp. Hardback, £29.99/US\$55 (approx.)/€45

(approx.).

ISBN 0-19-517432-1

..........

Cristina Rusu

European Journal of Human Genetics (2007) 15, 906;

doi:10.1038/sj.ejhg.5201861

$\mathrm{I}$ $\mathrm{t}$ was a great pleasure reviewing this book owing to its practical and comprehensive way of presenting such a complex material, reflecting 40 years' experience in the field of genetics, biochemistry and inborn errors of metabolism. The book is addressed to those interested in molecular mechanisms underlying embryonic nervous system development, as well as mechanisms that lead to structural and functional brain abnormalities.

The material is structured in 10 chapters covering different aspects of mental retardation and developmental delay. All chapters are very dense, starting with a few clinical data, followed by detailed, yet comprehensive information regarding molecular mechanisms for the most important specific disorders. The text is appropriately illustrated with schematic diagrams, which make it very easy to follow.

The introductory part discusses the key events in understanding the causes of mental retardation - all the social, environmental, metabolic and genetic factors are presented.
Neurogenesis and neuronal migration, maturation and function are discussed from the molecular point of view in the second chapter. Although the subject seems difficult, the author has achieved an outstanding presentation of the mechanisms underlying normal and abnormal development for each step separately.

The subsequent four chapters deal with the main categories of defects associated with mental retardation: structural brain anomalies, dysmorphic features (specific syndromes), other neurological defects (eg seizures) and metabolic defects. Each of them is a state of the art presentation in the field. I was very pleased to discover that some of the most recently described disorders (eg Mowat-Wilson's syndrome) have been included in the presentation, as well as the newest molecular data for every category of mental retardation, reflecting the vast experience of the author. The text combines molecular biology, genetics, metabolic pathways and clinical practice, but the focus on molecular data is more than obvious. The rich selection of illustrations (metabolic reac-
Dr P Macleod is at Pathology, Division of Medical Genetics, Victoria General Hospital, 1 Hospital Way, Victoria, British Columbia V8Z6R5, Canada. Tel: + 12507274461 ; Fax: + 1250727 4295; E-mail: patrick.macleod@viha.ca

tions and tables) makes the material practical and instructive. Once you start reading this book, you cannot put it away you become more and more eager to discover something new about the next disorder.

Autism is another part to be mentioned, especially because this is such a difficult field to manage in practice. The complex data regarding autism inheritance are clearly and simply presented, showing once more the experience of the author.

The chapter referring to functional genomics and epigenetics is remarkable. Many of the notions explained here have been recently introduced and not fully understood, but the author presents them in a very clear and comprehensive way.

Practitioners should not miss the last two chapters, discussing the necessary information for a correct diagnosis, prevention and treatment directions. They will appreciate the global assessment recommendations to establish the diagnosis, as well as the multitude of therapeutic approaches presented here.

Another particularly useful aspect for quick reference is the 'Index' - detailed and accurate - very good, when you look for a specific gene or metabolic pathway.

The bibliography is remarkable - 53 pages containing almost 1000 articles, most of them recent and very interesting.

In conclusion, this is an excellent book that should be on all pediatric and neurologic wards, medical genetics centers as well as medical school libraries. The material is dense, but provides you with the most updated data in the field

Cristina Rusu is at University of Medicine and Pharmacy, Medical Genetics, Str Universitatii 16, Iasi, Isi 700115, Romania. E-mail: abcrusu@gmail.com 